Pacific Journal of Mathematics

DISCONJUGACY OF A SELF-ADJOINT DIFFERENTIAL 


\title{
DISCONJUGACY OF A SELF-ADJOINT DIFFERENTIAL EQUATION OF THE FOURTH ORDER ${ }^{1}$
}

\author{
JOHN H. BARRETT ${ }^{2}$
}

Introduction. In a recent paper [10] W. Leighton and Z. Nehari investigated oscillation properties of solutions of self-adjoint differential equations of the fourth order

$$
\left(r(x) y^{\prime \prime}\right)^{\prime \prime}+\left(q(x) y^{\prime}\right)^{\prime}+p(x) y=0
$$

with particular attention to the cases where the middle term is missing, $r(x)>0$ and $p(x)$ does not change sign. In the present paper one of these particular cases

$$
\left(r(x) y^{\prime \prime}\right)^{\prime \prime}-p(x) y=0
$$

$(r(x)$ and $p(x)$ positive and continuous on $[a, \infty))$ will be pursued further with the object of paralleling the known theory of second order equation

$$
\left(r(x) y^{\prime}\right)^{\prime}+p(x) y=0
$$

with positive and continuous coefficients (e.g., see [2] and [12]). With only occasional minor modifications the terminology of [10], together with the fundamental properties of (1) established there, will be assumed throughout this paper. One point of departure is the distinction between "disconjugacy" and "non-oscillation" as the author has used them previously [2] for equation (2) in discussions which will be extended here to the fourth-order equation (1). It will be said that equation (1) is

(i) disconjugate if no nontrivial solution has more than 3 zeros on $[a, \infty)$ and, hence, no conjugate pairs exist on $[a, \infty)$ in the sense of Leighton and Nehari [10],

(ii) oscillatory if there is a nontrivial solution with infinitely many zeros on $[a, \infty)$.

(iii) nonoscillatory if every nontrivial solution has at most a finite number of zeros on $[a, \infty)$.

Recently, W. J. Coles [5] has developed Wirtinger-type inequalities in relation to the higher order equation

Received October 20, 1959.

1 This work was sponsored by the Office of Ordnance Research, U. S. Army, Contract DA-04-495-ORD-1088 with the University of Utah. Presented to the Amer. Math. Soc., January, 1960 .

2 Member, Mathematics Research Center, U. S. Army, Madison, Wisconsin for the 195960 academic year. 


$$
\left(r(x) y^{(m)}\right)^{(m)}+(-1)^{m+1} p(x) y=0 \quad(m=1,2,3, \cdots)
$$

by use of his Riccati systems [4] and in this discussion are included various sets of two-point boundary conditions, one of which is analogous to the well-known focal-point conditions for the second order equation (1) (see [2], [12] and [13]).

Again following the second-order discussions [2], associate with (1) its "reciprocal" equation [10, p. 369]

$$
\left(y^{\prime \prime} / p(x)\right)^{\prime \prime}-(1 / r(x)) y=0
$$

as was done for (2) with

$$
\left(y^{\prime} / p(x)\right)^{\prime}+(1 / r(x)) y=0^{3} .
$$

Note that $y(x)$ is a solution of (1) if, and only if,

$$
y_{1}(x)=r(x) y^{\prime \prime}(x)
$$

is a solution of $\left(1^{*}\right)$. Throughout this paper, the subscript "1" on a solution will stand for the leading coefficient times the second derivative of the solution.

In the first section known second-order definitions and theorems will be listed, which will be shown to be true almost verbatim for the fourth order case in the second section. The third section contains results following from Wirtinger-type inequalities, which are the fourth-order special cases of the above-mentioned results of Coles, and an extension of the eigenvalue discussion of Leighton and Nehari. The last section contains Coles' general theorem with minor modifications, as utilized in the preceding sections.

1. The second-order case. Consider equation (2) with the stated conditions on its coefficients [2].

DEFINITION 1.1. If a nontrivial solution of (2) satisfies the two-point boundary conditions $y(a)=y(b)=0, a<b$, then the smallest such number $b$ is designated as $\eta_{1}(a)$ and is called the first (right) conjugate point of $a$. If no such solution and number $b$ exist then equation (2) is said to be disconjugate.

DEFINITION 1.2. If a nontrivial solution of (2) satisfies $y(a)=y^{\prime}(b)=0$, $a<b$, then the smallest such number $b$ is designated by $\mu_{1}(a)$ and it is said that $a$ is the first (left) focal point of $b=\mu_{1}(a)$. The first two theorems are almost trivial for (2) but their counterparts for (1) require some proof, as is seen in the next section.

${ }^{3}$ Previously utilized by Leighton for boundedness theorems in [8]. 
Theorem 1.1. If $\eta_{1}(a)$ exists then so does $\mu_{1}(a)$ and $a<\mu_{1}(a)<\eta_{1}(a)$. Furthermore, if $\left(^{*}\right)$ denotes the same notation for the reciprocal equation $\left(2^{*}\right)$ then the existence of $\eta_{1}(a)$ implies, also, the existence of $\mu_{1}^{*}(a)$. In other words, if either $\eta_{1}(a)$ or $\eta_{1}^{*}(a)$ exist then both of $\mu_{1}(a)$ and $\mu_{1}^{*}(a)$ exist.

THEOREM 1.2. If $\mu_{1}(a)$ does not exist then for every solution $y(x)$ of (2), for which $y(a)=0$ and $y^{\prime}(a)>0$, it follows that $y(x)>0$ and $y^{\prime}(x)>0$ on $(a, \infty)$.

THEOREM 1.3. [2, p. 554] If $\mu_{1}(a)$ exists and $\int^{\infty}(1 / r)=\infty$ then $\eta_{1}(a)$ exists. Furthermore, for any solution $y(x)$ of $(2)$, for which $y^{\prime}(b)=0$, $y(b) \neq 0, a \leqq b$, it follows that $y(x)$ has a zero on $(b, \infty)$.

This theorem is due to Hille for $r \equiv 1$ and was utilized by Nehari [12]. It is noted that disconjugacy of (2) implies the non-existence of $\mu_{1}(a)$, if $\int^{\infty}(1 / r)=\infty$. Recall (e.g., [2]) that if $\int^{\infty}(1 / r)<\infty$ then $\mu_{1}(a)$ can exist even though $\eta_{1}(a)$ does not-in particular, when $\int^{\infty} p=\infty$.

THEOREM 1.4. [7] If $\int^{\infty}(1 / r)=\infty$ and $\int^{\infty} p=\infty$ then $\eta_{1}(a)$ exists and, in fact, equation (2) is oscillatory (for this result $p(x)$ may change sign).

The well-known relation of the focal-point problem to quadratic functionals ${ }^{4}$ was reiterated recently by W.T. Reid [13], when he gave a concise, self-contained development with applications to new oscillation criteria of (2).

THEOREM 1.5. [2,13]. If the number $\mu_{1}(a)$ does not exist then the quadratic functional

$$
I_{2}[u ; b]=\int_{a}^{b}\left(r\left(u^{\prime}\right)^{2}-p u^{2}\right)
$$

is strictly positive for every $b>a$ and every function $u(x)$ such that $u(x)$ is absolutely continuous, $u^{\prime} \in L_{2}(a, b)$ and $u$ has a zero of at least order one at $x=a$. This conclusion can also be stated as a Wirtingertype inequality

$$
\int_{a}^{b} p u^{2}<\int_{a}^{b} r\left(u^{\prime}\right)^{2}
$$

2. The fourth-order case. Consider the equations

$$
\left(r(x) y^{\prime \prime}\right)^{\prime \prime}-p(x) y=0,
$$

${ }^{4}$ For the general classical theory see Morse [11] and for the theory for singular functionals see Leighton [9]. 
$(r(x)$ and $p(x)$ positive and continuous on $[a, \infty))$, and

$$
\left(y^{\prime \prime} / p(x)^{\prime \prime}-y / r(x)=0\right. \text {. }
$$

The following conjugate point definition is that of Leighton and Nehari [10].

DEFINITION 2.1. If a non-trivial solution of (1) satisfies the two-point boundary conditions

$$
y(a)=y^{\prime}(a)=y(b)=y^{\prime}(b)=0, a<b,
$$

then the smallest such number $b$ is designated by $\eta_{1}(a)$ and is called the first (right) conjugate point of $a$. Recall from [10] that such a number exists if (1) has a nontrivial solution which has double zeros at $a$ and $\eta_{1}(a)$, is non-zero on $\left(a, \eta_{1}(a)\right)$ and any essentially different (linearly independent) solution of (1) has at most 3 zeros on $\left[a, \eta_{1}(a)\right]$.

DeFinition 2.2. If a nontrivial solution of (1) satisfies

$$
\begin{gathered}
y(a)=y^{\prime}(a)=y_{1}(b)=y_{1}^{\prime}(b)=0, a<b, \\
\left(\text { recall } y_{1}=r y^{\prime \prime}\right)
\end{gathered}
$$

then the smallest such number $b$ is designated by $\mu_{1}(a)$. The solutions of (1) which are particularly useful in the following analysis are those whose Wronskian at $x=a$ is

$$
\begin{array}{lllll} 
& y & y^{\prime} & y_{1} & y_{1}^{\prime} \\
y=U(x): & 1 & 0 & 0 & 0 \\
y=V(x): & 0 & 1 & 0 & 0 \\
y=u(x): & 0 & 0 & 1 & 0 \\
y=v(x): & 0 & 0 & 0 & 1
\end{array}
$$

By [10, Lemma 2.1] all of $y, y^{\prime}, y_{1}$ and $y_{1}^{\prime}$ for $y=U, V, u$ and $v$ are positive on $(a, \infty)$.

Lemma 2.1. [10] If $y(x)$ and $z(x)$ are solutions of (1) then

$$
S[y ; z]=y z_{1}^{\prime}-z y_{1}^{\prime}-y^{\prime} z_{1}+z^{\prime} y_{1} \equiv C, \text { a constant. }
$$

In [10] the non-self-adjoint form of the following is established and utilized in establishing conjugate point (oscillation) theorems.

LEMmA 2.2. If $y(x)$ and $z(z)$ are solutions of (1) such that $S[y ; z]=0$ and $y(x) \neq 0$ on $I \subset[a, \infty)$ then $W(x)=y z^{\prime}-z y^{\prime}$ satisfies the secondorder self-adjoint equation

$$
\left(r W^{\prime} / y^{2}\right)^{\prime}+\left(2 y_{1} / y^{3}\right) W=0 \text { on } I .
$$


Note that if $y>0$ and $y_{1}^{\prime}>0$ on $I$ (such solutions exist for any such $I$ ) both coefficients in (8) are positive and the results of the first section apply. The following is an example of the importance of (8):

LEMma 2.3. [10. Th. 3.11] If $y(x)$ is a solution of (1) having at most a finite number of zeros then (1) is oscillatory if, and only if, (8) is oscillatory.

The present discussion will be concerned with relations between the last two solutions, $u(x)$ and $v(x)$, defined by (7), since every solution of (1) having a double zero at $x=a$ can be expressed by

$$
y=y_{1}(a) u(x)+y_{1}^{\prime}(a) v(x) \text {. }
$$

As in [10], note that in order for any nontrivial solution of (1) to have both a double zero at $x=a$ and at $x=b$ (i.e., satisfy boundary conditions (5)) it is necessary and sufficient that

$$
\sigma(x)=u(x) v^{\prime}(x)-u^{\prime}(x) v(x)
$$

vanish at $x=b$. Furthermore, $\eta_{1}(a)$ (of Definition 2.1) is the smallest such $b>a$. Observe further that in order for a nontrivial solution of (1) to satisfy the conditions (6) it is necessary and sufficient that

$$
\rho(x)=u_{1}(x) v_{1}^{\prime}(x)-u_{1}^{\prime}(x) v_{1}(x)
$$

vanish at $x=b$. The stage is now set for the verbatim fourth-order analog of Theorem 1.1.

THEOREM 2.1. If $\eta_{1}(a)$ exists then so does $\mu_{1}(a)$ and $a<\mu_{1}(a)<\eta_{1}(a)$. Furthermore, if $\left(^{*}\right)$ denotes the same notation for the reciprocal equation $\left(^{*}\right)$ then $\eta_{1}(a)$ implies, also, the existence of $\mu_{1}^{*}(a)$.

Proof. Let $Z(x)$ be a solution of (1) having double zeros at $x=a$ and $x=\eta_{1}(a)$ and positive in $\left(a, \eta_{1}(a)\right)$. There exist inflection points $x_{1}<x_{2}$ of $y=Z(x)$ on $\left(a, \eta_{1}(a)\right)$ such that $Z^{\prime \prime}\left(x_{1}\right)=Z^{\prime \prime}\left(x_{2}\right)=0$ and $Z^{\prime \prime}<0$ on $\left(x_{1}, x_{2}\right)$. Recall that $u(x)$ is the solution of (1) which satisfies

$$
u(a)=u^{\prime}(a)=u_{1}^{\prime}(a)=0, u_{1}(a)=1 .
$$

By use of a fundamental technique of [10], since $\left(Z^{\prime \prime} / u^{\prime \prime}\right)^{\prime}$ must change sign on $\left(x_{1}, x_{2}\right)$, say at $x=b$. There exists a number $\lambda$ such that the solution $y=Z(x)-\lambda u(x)$ satisfies the boundary conditions (6) and $a<\mu_{1}(a) \leqq b<\eta_{1}(a)^{5}$. For the second part consider the pair of solutions $U, u$. Since $S[u ; v]=0$ then $\sigma(x)=u v^{\prime}-v u^{\prime}$ is a solution of

5 This result may also be established easily by means of an indirect argument using equation $\left(8_{1}\right)$ following. 
$\left(8^{\prime}\right)$

$$
\left(\frac{r \sigma^{\prime}}{u^{2}}\right)^{\prime}+\frac{2 u_{1}}{u^{3}} \sigma=0 \text { and }\left(\frac{r \sigma^{\prime}}{v^{2}}\right)^{\prime}+\frac{2 v_{1}}{v^{3}} \sigma=0 \text { on }(a, \infty)
$$

Note that $x=a$ is a singular point. Similarly, $S[U, u]=0$ and $\sigma_{1}(x)=$ $U u^{\prime}-u U^{\prime}$ is a solution of $\left(8^{\prime}\right)$ and

$$
\left(\frac{r \sigma_{1}^{\prime}}{U^{2}}\right)^{\prime}+\frac{2 U_{1}}{U^{3}} \sigma_{1}=0 \text { on }[a, \infty) \text {. }
$$

Finally, $S[U, V]=0$ and $\sigma_{2}=U V^{\prime}-V U^{\prime}$ is a solution of $\left(8^{\prime \prime}\right)$. Since $\sigma(a)=0=\sigma\left(\eta_{1}\right)=\sigma_{1}(a)$ and $\sigma_{2}(a)>0, \sigma_{2}^{\prime}(a)=0$, then simple comparison techniques give that $\sigma_{1}$ has a zero $x_{1}$ on $\left(a, \eta_{1}\right)$ and that $\sigma_{2}(x)$ has a zero $x_{2}$ on $\left(a, x_{1}\right) \subset\left(a, \eta_{1}\right)$. Because of the relationship between (1) and $\left(1^{*}\right)$ it is easily seen that the smallest such number $x_{2}$ is actually $\mu_{1}^{*}(\alpha)$ and the theorem is proved.

While Theorem 1.2 is not true for (1) a similar theorem does hold.

THEOREM 2.2. If $\mu_{1}(a)$ does not exist then there exists a solution $y(x)$ such that $y(a)=y^{\prime}(a)=0, y(x)>0, y^{\prime}(x)>0 \quad y_{1}(x)>0$ and $y_{1}^{\prime}(x)<0$, on $(a, \infty)$.

The proof will be accomplished by two lemmas concerning the ratios involving the particular solutions $u(x)$ and $v(x)$ of (7).

$$
\lambda_{0}=u / v, \lambda_{1}=u^{\prime} / v^{\prime}, \lambda_{2}=u_{1} / v_{1}, \lambda_{3}=u_{1}^{\prime} / v_{1}^{\prime} .
$$

LEMMA 2.4. If $\mu_{1}(a)$ does not exist then

$$
\lambda_{0}>\lambda_{1}>\lambda_{2}>\lambda_{3}>0 \text { on }(a, \infty) .
$$

Proof of lemma. That all $\lambda_{i}$ are positive is obvious. Also, if Lemma 2.2 is applied to $\left(1^{*}\right)$ and its solutions $u_{1}$ and $v_{1}$ then $\rho(x)$, defined by (10), satisfies

$$
\begin{array}{rll}
\left(\rho^{\prime} / p u_{1}^{2}\right)^{\prime}+2 u \rho / u_{1}^{3}=0 & \text { on } \quad[a, \infty) \\
\left(\rho^{\prime} / p v_{1}^{2}\right)^{\prime}+2 v \rho / v_{1}^{3}=0 & \text { on } \quad(a, \infty) .
\end{array}
$$

Note that $x=a$ is not a singular point of the first equation of $\left(8_{1}\right)$. The following useful relations are derived by routine calculations:

$$
\left\{\begin{array}{l}
\lambda_{0}-\lambda_{1}=\sigma / v v^{\prime} \\
\lambda_{1}-\lambda_{2}=\tau / v^{\prime} v_{1} \\
\lambda_{2}-\lambda_{3}=\rho / v_{1} v_{1}^{\prime}
\end{array}\right.
$$

$$
\left\{\begin{aligned}
\rho^{\prime} / p & =-r \sigma^{\prime} \\
\left(r \sigma^{\prime}\right)^{\prime} & =2 \tau
\end{aligned}\right.
$$

where

$$
\tau(x)=u^{\prime} v_{1}-v^{\prime} u_{1} \equiv u v_{1}^{\prime}-v u_{1}^{\prime},
$$

the latter identity following from the fact that $S[u ; v]=0$.

Since $\mu_{1}(a)$ does not exist and $\rho(a)=1$ then $\rho(x)>0$ on $[a, \infty)$. Also, 
$\rho^{\prime}(a)=0$ and, hence, integration of the first equation of $\left(8_{1}\right)$ gives $\rho^{\prime}(x)<0$ on $(a, \infty)$. Therefore, $\sigma^{\prime}(x)>0$ and $\sigma(x)>0$ on $(a, \infty)$. In order to show that $\tau(x)>0$, recall that $\left(\rho^{\prime} / p\right)^{\prime}=-2 \tau$ and from the second equation of $\left(8_{1}\right)$ :

$$
2 \tau / v_{1}^{2}=2 v \rho / v_{1}^{3}-2 v_{1}^{\prime} \rho^{\prime} / p v_{1}^{3}>0 \text { on }(a, \infty) .
$$

Therefore, all of the differences (12) are positive on $(a, \infty)$.

LEMmA 2.5. If $\mu_{1}(a)$ does not exist then $\lambda_{0}, \lambda_{1}$ and $\lambda_{2}$ are decreasing functions and $\lambda_{3}$ is an increasing function on $(a, \infty)$.

Proof of lemma. Simple calculations yield

$$
\lambda_{0}^{\prime}=-\sigma / v^{2}, \lambda_{1}^{\prime}=-\tau / r\left(v^{\prime}\right)^{2}, \lambda_{2}^{\prime}=-\rho / u_{1}^{2}, \lambda_{3}^{\prime}=p \tau /\left(v_{1}^{\prime}\right)^{2}
$$

from which the result follows immediately.

To complete the proof of Theorem 2.2, let $\lambda^{*}$ be a positive number such that

$$
\lambda_{0}(x)>\lambda_{1}(x)>\lambda_{2}(x)>\lambda^{*}>\lambda_{3}(x) \text { on }(a, \infty) .
$$

Then $y\left(x, \lambda^{*}\right)=u(x)-\lambda^{*} v(x)$ satisfies the required conditions.

An example of (1) for which $\mu_{1}(a)$ does not exist is $\left(e^{-2 x} y^{\prime \prime}\right)^{\prime \prime}-e^{-2 x} y=0$ for which direct calculations show that $\rho(x)>0$ on $(a, \infty)$. This example should be compared with the similar one for the second-order case [2].

THEOREM 2.3. If $\mu_{1}(a)$ exists and $\int^{\infty}(1 / r)=\infty$ then $\eta_{1}(a)$ exists.

Proof. In addition to the identities (12), (13) and (14) note that

$$
\left\{\begin{array}{l}
\lambda_{0}-\lambda_{2}=r \sigma^{\prime} / v v_{1} \\
\lambda_{0}-\lambda_{3}=\tau / v v_{1}^{\prime} \\
\lambda_{1}-\lambda_{3}=\tau^{\prime} / v^{\prime} v_{1}^{\prime}
\end{array}\right.
$$$$
\text { (16) }\left\{\begin{array}{l}
\tau^{\prime}=\left(u^{\prime} \tau-\sigma u_{1}^{\prime}\right) / u \\
\tau^{\prime}=\left(u^{\prime} \rho+\tau u_{1}^{\prime}\right) / u_{1} \\
\tau^{\prime \prime}=\frac{1}{r} \rho-p \sigma \quad \text { or } \\
\left(r \sigma^{\prime}\right)^{\prime \prime \prime}+2 p \sigma=2 \rho / r .
\end{array}\right.
$$

and that $\tau(x)$ satisfies the second order self-adjoint equation with positive coefficients:

$$
\left(\frac{\tau^{\prime}}{\sqrt{u^{\prime} u_{1}^{\prime}}}\right)^{\prime}+\frac{1}{2 \sqrt{u^{\prime} u_{1}^{\prime}}}\left(\frac{u_{1}^{\prime}}{r u^{\prime}}+\frac{p u^{\prime}}{u_{1}^{\prime}}\right) \tau=0 \quad \text { on } \quad(a, \infty) .
$$

Assume that the theorem is not true, i.e., (1) is disconjugate. Thus $\sigma(x)>0$ on $(a, \infty)$ and since $\int^{\infty}\left(u^{2} / r\right)=\infty$, Theorem 1.3 (second part) may be applied to 


$$
\left(r \sigma^{\prime} / u^{2}\right)^{\prime}+2 u_{1} \sigma / u^{3}=0 \text { on }(a, \infty) .
$$

to obtain that $\sigma^{\prime}(x)>0$ on $(a, \infty)$. Hence $\rho^{\prime}(x)<0$ and $\rho(x)$ has only one zero, namely $\mu_{1}(a)$, on $(a, \infty)$. Since

$$
\begin{array}{ccc}
(\tau / u)^{\prime}=-\sigma u_{1}^{\prime} / u^{2}<0 & \text { on } & (a, \infty), \lim _{x \rightarrow a}(\tau / u)=\frac{1}{2}>0, \\
\left.\tau(a)=\tau^{\prime} a\right)=0 & \text { and } \tau^{\prime \prime}(a)>0 .
\end{array}
$$

Then $\tau(x)$ can have at most one zero on $(a, \infty)$. Suppose first that such a zero $x=t_{1}$ exists. Then $\tau(x)>0$ on $\left(\alpha, t_{1}\right)$ and $\tau(x)<0$ on $\left(t_{1}, \infty\right)$. Note that $\int^{\infty} \sqrt{u^{\prime} u_{1}^{\prime}}=\infty$ and since (17) is disconjugate, Theorem 1.3 guarantees that its solution $\tau(x)$ has only one point $x=t_{1}^{\prime}$ on $\left(\alpha, t_{1}\right)$ where $\tau^{\prime}=0$ and $\tau^{\prime}<0$ on $\left(t_{1}^{\prime}, \infty\right)$. The first equation of (16) yields that $a<\mu_{1}<t_{1}^{\prime}<t_{1}$.

On the half-line $\left(t_{1}, \infty\right)$ equations (12), (14) and (15) yield

$$
\begin{gathered}
\lambda_{3}(x)>\lambda_{0}(x)>\lambda_{2}(x)>\lambda_{1}(x) \\
\lambda_{3}^{\prime}<0, \lambda_{0}^{\prime}<0, \lambda_{2}^{\prime}>0, \lambda_{1}^{\prime}>0 .
\end{gathered}
$$

Because of the above monotonicity there exists a positive constant $\lambda^{*}$ such that on $\left(t_{1}, \infty\right)$,

$$
\lambda_{3}>\lambda_{0}>\lambda^{*}>\lambda_{2}>\lambda_{1} .
$$

Let $y(x)=u(x)-\lambda^{*} v(x)$, a solution of (1). Then $y(x)>0, y^{\prime}(x)<0$ and $y^{\prime \prime}(x)<0$ (since $\left.y_{1}<0\right)$ on $\left(t_{1}, \infty\right)$, which is contradictory information.

Therefore, $\tau(x)>0$ on $(a, \infty)$ and by Theorem 1.3, $\tau^{\prime}(x)>0$. Thus on the half-line $\left(\mu_{1}, \infty\right)$ :

$$
\begin{gathered}
\lambda_{0}(x)>\lambda_{1}(x)>\lambda_{3}(x)>\lambda_{2}(x) \\
\lambda_{0}^{\prime}<0, \lambda_{1}^{\prime}<0, \lambda_{3}^{\prime}>0, \quad \lambda_{2}^{\prime}>0 .
\end{gathered}
$$

As above there exists a positive constant $\lambda^{*}$ between $\lambda_{1}$ and $\lambda_{3}$ on $\left(\mu_{1}, \infty\right)$.

For $y(x)=u(x)-\lambda^{*} v(x)$ on the interval $\left(\mu_{1}, \infty\right)$ :

$$
y(x)>0, y^{\prime}(x)>0, y_{1}(x)<0 \text { and } y_{1}^{\prime}(x)>0
$$

which is contradictory, using that $\int^{\infty}(1 / r)=\infty$.

THEOREM 2.4. If $\int^{\infty}(1 / r)=\infty$ and $\int^{\infty} p u_{1}^{2}=\infty$ then $\eta_{1}(a)$ exists and, in fact, equation (1) is oscillatory.

The crucial point of the proof is the following which follows immediately by application of Theorem 1.3 to equation $\left(8_{1}\right)$.

LEMMA 2.6. If $\int^{\infty} p u_{1}^{2}=\infty$ then $\mu_{1}(a)$ exists. 
Proof of theorem. $\int^{\infty} p u_{1}^{2}=\infty$ implies that $\mu_{1}(a)$ exists which, together with $\int^{\infty}(1 / r)=\infty$ and Theorem 2.3 gives that $\eta_{1}(a)$ exists. Since for any $a_{1}>a, \eta_{1}\left(a_{1}\right)$ exists, then by [10] equation (1) is oscillatory.

Because of the monotonicity of $u(x)$ and $u_{1}(x)$ it follows that

Corollary 2.4.1. If $\int^{\infty}(1 / r)=\infty$ and $\int^{\infty} p=\infty$ then (1) is oscillatory.

Further corollaries are obtained by a more careful examination of the properties of $u(x)$ and its derivatives. Integration of (1) and consideration of the initial conditions for $u(x)$ yield

$$
u_{1}(x)=1+\stackrel{x}{\mathrm{I}}_{a}^{2}(p u) \text { and } u(x)=\stackrel{x}{\mathrm{I}}_{a}^{2}\left(u_{1} / r\right),
$$

where the Riemann-Liouville notation is used for the iterated integrals. For $a<x_{0} \leqq x<\infty$ it follows that

$$
\int_{x_{0}}^{\infty}\left(u^{2} / r\right)>u_{1}^{2}\left(x_{0}\right) \int_{x_{0}}^{\infty}(1 / r(x))\left[{ }_{x_{0}}^{x}(1 / r)\right]^{2} d x
$$

and

$$
\begin{gathered}
\int_{x_{0}}^{\infty} p u_{1}^{2}>\int_{x_{0}}^{\infty} p(x)\left[1+u\left(x_{0}\right) \underset{x_{0}}{\stackrel{x}{2} p]^{2} d x}\right. \\
=\int_{x_{0}}^{\infty} p+2 u\left(x_{0}\right) \int_{x_{0}}^{\infty}\left(p(x)\left({ }_{x_{0}}^{x} p\right) d x+u^{2}\left(x_{0}\right) \int_{0}^{\infty} p(x)\left({\stackrel{x}{\mathrm{I}_{0}} p}_{x_{0}}^{2} p\right)^{2} d x .\right.
\end{gathered}
$$

COROLlary 2.4.2. If $\int^{\infty}(1 / r(x)) d x=\infty$ and $\int^{\infty} p(x)\left({\stackrel{\mathrm{I}}{\mathrm{I}_{0}}}^{2} p\right)^{2} d x=\infty$ then (1) is oscillatory.

Note that for $a<x_{0}<x_{1} \leqq x<\infty,{ }_{x_{0}}^{x} p \geqq\left(x-x_{1}\right) \int_{x_{0}}^{x_{1}} p \rightarrow \infty$ as $x \rightarrow \infty$ and the following result of Nehari and Leighton follows.

CoROLlaRY 2.4.3. $\quad$ [10, Th. 6.8] If $r(x) \leqq m$ and (1) is nonoscillatory then $\int^{\infty} x^{2} p(x) d x<\infty$.

In connection with the above oscillation theorems it is appropriate to list two known theorems insuring nonoscillation.

Theorem [10, Th. 6.12] If $P(x)=\stackrel{\infty}{3}_{x} p$ and $\int^{\infty}(P / r)<\infty$ then (1) is non-oscillatory.

Theorem [10, Th. 6.11] If $\int^{\infty} 1 / r<\infty$ and $\int_{x}^{\infty} x^{2} p(x) d x<\infty$ then (1) is non-oscillatory.

${ }^{6}$ Also, a special case of a more general result for fourth-order systems by Sternberg and Sternberg [14]. 
3. An eigenvalue problem and Wirtinger-type inequalities. Leighton and Nehari have shown [10, Th. 6.6 and 6.7] that equation (1) is disconjugate on $[a, \infty)$ if, and only if, the least eigenvalue $\lambda(b)$ of the “conjugate-point" problem

$$
\left(r y^{\prime \prime}\right)^{\prime \prime}-\lambda p y=0, y(a)=y^{\prime}(a)=y(b)=y^{\prime}(b)=0 .
$$

satisfies $\lambda(b)>1$ for all $b>a$. Furthermore, if equation (1) is disconjugate on $[a, \infty)$ then $I_{4}[w ; b] \geqq 0$ (see equation (25)) for all $w(x)$ of class $D^{\prime \prime}(a, b) \in L_{2}(a, b)$ which satisfy $w(a)=w^{\prime}(a)=w(b)=w^{\prime}(b)=0$. Finally, in the spirit of [12] they obtained a number of nonoscillation theorems by taking special examples of such $w(x)$.

Consider here the "focal-point" problem as for the second-order case in $[2,12]$.

$$
\left(r y^{\prime \prime}\right)^{\prime \prime}-\lambda p y=0, y(a)=y^{\prime}(a)=y_{1}(b)=y_{1}^{\prime}(b)=0 .
$$

For each $b>a$ let $\lambda_{1}(b)$ be the least eigenvalue and $y=z(x)$ be a corresponding eigenfunction. Integration by parts gives

$$
\int_{a}^{b} p z^{2}=\lambda_{1}(b) \int_{a}^{b} r\left(z^{\prime \prime}\right)^{2} .
$$

If there does not exist a number $\mu_{1}(a)$ then $\lambda=1$ is not an eigenvalue for any $b>a$ and by Theorem 2.2, there exists a solution $y(x)$ of (1) for which $y(a)=y^{\prime}(a)=0$ and $y(x)>0, y^{\prime}(x)>0, y_{1}(x)>0, y_{1}^{\prime}(x)<0$ on $(a, \infty)$. This is but a special case of the general theorem of Coles [5], as will be seen in the last section, and it follows ${ }^{7}$ that for every $b>a$

$$
\int_{a}^{b} p u^{2}<\int_{a}^{b} r\left(u^{\prime \prime}\right)^{2} \text {, i.e., } I_{4}[u ; b]>0
$$

for every function $u(x)$ for which $u^{\prime}$ is absolutely continuous, $u^{\prime \prime} \in L_{2}(a, b)$ and $u$ has a double zero at $x=a$.

Lemma 3.1. The number $\mu_{1}(a)$ does not exist if, and only if, the eigenvalue $\lambda_{1}(b)>1$ for all $b>a$.

Proof. If $\mu_{1}(a)$ exists then for $b=\mu_{1}(a), \lambda\left(\mu_{1}(a)\right)=1$. If $\mu_{1}(a)$ does not exist then (20) and (21) yield that $\lambda_{1}(b)>1$ for $b>a$ and the lemma is proved.

By combining the above lemma with Theorem 2.3 it follows that (recall the special monotone solution $u(x)$ of $(1)$ ):

THEOREM $3.1^{8}$. If $\int^{\infty}(1 / r)=\infty$ then equation $(1)$ is disconjugate on

7 See concluding statement of section 4 .

8 This result has also been obtained by H. C. Howard by means of Rayleigh quotients in his dissertation at Carnegie Institute of Technology, June 1958; to appear in the Transactions of the American Mathematical Society 96 (1960), 296-311. 
$[a, \infty)$ if, and only if, $\lambda_{1}(b)>1$ for all $b>a$ and if $(1)$ is disconjugate then for every $b>a$

$$
\int_{a}^{b} p w^{2}<\int_{a}^{b} r\left(w^{\prime \prime}\right)^{2}
$$

for all $w(x)$ such that $w^{\prime}(x)$ is absolutely continuous, $w^{\prime \prime} \in L_{2}(a, b)$ and $w(x)$ has a double zero at $x=a$.

Note that for such coefficients as $r \equiv 1$ or $\int^{\infty}(1 / r)=\infty$ the Wirtinger-type inequality requires a double zero only at $x=a$ while the result stated at the beginning requires double zeros at both $x=a$ and $x=b$.

An application of Theorem 3.1 to the reciprocal equation $\left(1^{*}\right)$ yields

COROLLARY 3.1.1. If $\int^{\infty} p=\infty$ and equation $\left(1^{*}\right)$ is disconjugate then for every $b>a$

$$
\int_{a}^{b} \frac{1}{r} w^{2}<\int_{a}^{b} \frac{1}{p}\left(w^{\prime \prime}\right)^{2}
$$

for the above class of functions $w$.

4. Higher order equations. The following is the theorem of Coles [5] which has been utilized several times in the preceding sections. It should be noted that his proof for the case $r \equiv 1$ carries over step-forstep for the following.

THEOREM C. If $m$ is a positive integer; $r(x)>0$ and $p(x)$ are both continuous on $[a, b], a<b$; and $y(x)$ is a solution of

$$
\left(r(x) y^{(m)}\right)^{(m)}-p(x) y=0 ; y_{1}(x)=r(x) y^{(m)}(x)
$$

such that

$$
\left\{\begin{aligned}
&(-1)^{m} p(x) y(x) \geqq 0 \text { but } \not \equiv 0 \text { on }[a, b] \\
& p_{i} y^{(m-i)}\left(c_{i}\right) \geqq 0 \quad(i=1,2, \cdots, m) \\
& q_{i} y_{1}^{(i)}\left(d_{i}\right) \geqq 0 \quad(i=0,1, \cdots, m-1)
\end{aligned}\right.
$$

where

$$
\begin{gathered}
k_{i}=0 \text { or } 1 \quad(i=0,1, \cdots, m) \text { such that } \sum_{i=0}^{m} k_{i} \text { is even } \\
c_{i}=\left\{\begin{array}{l}
a, k_{i}=0 \\
b, k_{i}=0
\end{array} \quad d_{i}=\left\{\begin{array} { l } 
{ a , k _ { i + 1 } = 0 } \\
{ b , k _ { i + 1 } = 0 }
\end{array} \quad \left\{\begin{array}{l}
c_{i}^{*}=a+b-c_{i} \\
d_{i}^{*}=a+b-d_{i}
\end{array}\right.\right.\right. \\
p_{i}=(-1)^{\Sigma_{j=0}^{i} k_{j}}, q_{i}=(-1)^{i} p_{i} \quad(i=0, \cdots, m-1)
\end{gathered}
$$

then 


$$
\begin{aligned}
p_{i} y^{(m-i)}(x)>0 \text { on }(a, b) \text { and at } c_{i}^{*} & (i=1,2, \cdots, m) \\
q_{i} y_{1}^{(i)}(x) \geqq 0 \text { on }[a, b] \text { and }>0 \text { at } d_{i}^{*} & (i=0,1, \cdots, m-1),
\end{aligned}
$$

and the last inequality is strictly positive if $p(x)$ is not identically zero on any subinterval of $[a, b]$. Furthermore, for every $b>a$

$$
I_{2 m}[u ; b]=\int_{a}^{b}\left[r\left(u^{(m)}\right)^{2}-(-1)^{m} p u^{2}\right]
$$

is non-negative for all functions $u(x)$ such that

$$
\left\{\begin{array}{l}
u^{(m-1)}(x) \text { is absolutely continuous } \\
u^{(m)} \in L_{2}[a, b] \text { and } u^{(i)}\left(d_{m-i-1}^{*}\right)=0
\end{array}\right.
$$

(of at least order 1)

for $i=0,1, \cdots, m-1$; with $I_{2 m}=0$ if, and only if, $u(x)$ is a constant times a solution of (22) which, in addition, satisfies

$$
q_{i} y^{(i)}\left(d_{i}\right)=0 \quad(i=0,1, \cdots, m-1) .
$$

Note that the special case in $\S 3$ is that for $m=2$ and $k_{1}=k_{2}=k_{3}=0$.

For this case, Coles' method reduces to the following: If $y(x)$ is a solution of (1) such that $y>0$ and $y^{\prime}>0$ on $[a, b]$ then by integrating by parts and completing squares

$$
\begin{aligned}
\int_{a}^{b} p u^{2}= & {\left[\frac{y_{1}^{\prime} u^{2}}{y}-\frac{y_{1}\left(u^{\prime}\right)^{2}}{y^{\prime}}\right]_{a}^{b} } \\
& +\int_{a}^{b} \frac{y_{1}^{\prime}}{y^{\prime}}\left[u^{\prime}-\frac{y^{\prime}}{y} u\right]^{2}-\int_{a}^{b} r\left[u^{\prime \prime}-\frac{y^{\prime \prime}}{y^{\prime}} u^{\prime}\right]^{2}+\int_{a}^{b} r\left(u^{\prime \prime}\right)^{2} .
\end{aligned}
$$

Using Theorem 2.2 the inequality (21) follows immediately.

\section{BIBLIOGRAPHY}

1. J. H. Barrett, Behavior of solutions of second-order self-adjoint differential equations, Proc. AMS 8 (1955), 510-518.

2. - Disconjugacy of second-order linear differential equations with non-negative coefficients, Proc. AMS 10 (1959), 552-561.

3. P. R. Beesack, Integral inequalities of the Wirtinger-type, Duke Math. J. 25 (1958), 477-498.

4. W. J. Coles, Linear and Riccati Systems, Duke Math. J. 22 (1955), 333-338.

5. —_ A general Wirtinger-type inequality, Duke. Math. J. 27 (1960), 133-138.

6. E. Hille, Non-oscillation Theorems, Trans. AMS 64 (1948), 234-252.

7. W. Leighton, On self-adjoint differential equations of second-order, J. London Math. Soc. 27 (1952), 37-47.

8. - Bounds for the solutions of a second order linear differential equation, Proc. Nat. Acad. Sci. 35 (1949), 190-191.

9. —_ Principal quadratic functionals, Trans. AMS 67 (1949), 253-274. 
10. W. Leighton and Z. Nehari, On the oscillation of solutions of self-adjoint linear differential equations of the fourth order Trans. AMS. 89 (1958), 325-377.

11. M. Morse, Calculus of Variations in the Large, New York, 1934.

12. Z. Nehari, Oscillation criteria for second-order linear differential equations, Trans. AMS 85 (1958), 428-445.

13. W. T. Reid, A comparison theorem for self-adjoint differential equations of second order, Annals of Math. 65 (1957), 197-202.

14. H. M. and R. L. Sternberg, A two-point boundary problem for ordinary self-adjoint differential equations of the fourth order, Canadian J. Math. 6 (1954), 416-419.

The UNIVERSity OF UTAH 



\section{PACIFIC JOURNAL OF MATHEMATICS}

\section{EDITORS}

Ralph S. Phillips

Stanford University

Stanford, California

F. H. BrownelL

University of Washington

Seattle 5 , Washington
A. L. Whiteman

University of Southern California Los Angeles 7. California

L. J. PAIGE

University of California

Los Angeles 24, California

ASSOCIATE EDITORS
E. F. BECKENBACH
D. DERRY
H. L. ROYDEN
E. G. STRAUS
T. M. CHERRY
M. OHTSUKA
E. SPANIER
F. WOLF

\section{SUPPORTING INSTITUTIONS}

UNIVERSITY OF BRITISH COLUMBIA

CALIFORNIA INSTITUTE OF TECHNOLOGY

UNIVERSITY OF CALIFORNIA

MONTANA STATE UNIVERSITY

UNIVERSITY OF NEVADA

NEW MEXICO STATE UNIVERSITY

OREGON STATE COLLEGE

UNIVERSITY OF OREGON

OSAKA UNIVERSITY

UNIVERSITY OF SOUTHERN CALIFORNIA
STANFORD UNIVERSITY

UNIVERSITY OF TOKYO

UNIVERSITY OF UTAH

WASHINGTON STATE COLLEGE,

UNIVERSITY OF WASHINGTON

AMERICAN MATHEMATICAL SOCIETY

CALIFORNIA RESEARCH CORPORATION

HUGHES AIRCRAFT COMPANY

SPACE TECHNOLOGY LABORATORIES

NAVAL ORDNANCE TEST STATION

Printed in Japan by International Academic Printing Co., Ltd., Tokyo, Japan

Reprinted 1966 in the United States of America 


\section{Pacific Journal of Mathematics}

\section{Vol. 11, No. $1 \quad$ November, 1961}

A. A. Albert, Generalized twisted fields ............................ 1

Richard Arens, Operational calculus of linear relations ................... 9

John Herbert Barrett, Disconjugacy of a self-adjoint differential equation of the fourth order ....................................... 25

Paul Richard Beesack, Hardy's inequality and its extensions ............... 39

Julius Rubin Blum and David Lee Hanson, On invariant probability measures.

II .............................................

Robert Allen Bonic, Symmetry in group algebras of discrete groups.......... 73

R. Creighton Buck, Multiplication operators ...................... 95

Jack Gary Ceder, Some generalizations of metric spaces ................. 105

Meyer Dwass, Random crossings of cumulative distribution functions ......... 127

Albert Edrei, Wolfgang H. J. Fuchs and Simon Hellerstein, Radial distribution and

deficiencies of the values of a meromorphic function ............... 135

William Cassidy Fox, Harmonic functions with arbitrary local singularities ..... 153

Theodore Thomas Frankel, Manifolds with positive curvature ............... 165

Avner Friedman, A strong maximum principle for weakly subparabolic

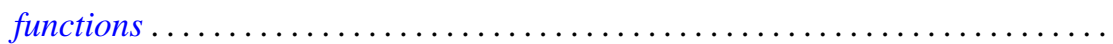

Watson Bryan Fulks and J. O. Sather, Asymptotics. II. Laplace's method for

multiple integrals ......................................

Adriano Mario Garsia and Eugene Richard Rodemich, An embedding of Riemann

surfaces of genus one ..................................... 193

Irving Leonard Glicksberg, Weak compactness and separate continuity......... 205

Branko Grünbaum, On a conjecture of H. Hadwiger .................. 215

Frank J. Hahn, On the action of a locally compact group on $E_{n} \ldots \ldots \ldots \ldots \ldots . . \ldots 221$

Magnus R. Hestenes, Relative hermitian matrices ..................... 225

G. K. Kalisch, On similarity invariants of certain operators in $L_{p} \ldots \ldots \ldots \ldots .247$

Yitzhak Katznelson and Walter Rudin, The Stone-Weierstrass property in Banach

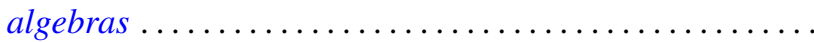

Samir A. Khabbaz, The subgroups of a divisible group $G$ which can be represented as intersections of divisible subgroups of $G \ldots \ldots \ldots \ldots \ldots \ldots \ldots \ldots \ldots . \ldots \ldots 7$

Marvin Isadore Knopp, Construction of a class of modular functions and forms .......................................... 275

Charles Alan McCarthy, Commuting Boolean algebras of projections .......... 295

T. M. MacRobert, Transformations of series of E-functions ................ 309

Heinz Renggli, An inequality for logarithmic capacities ................. 313

M. S. Robertson, Applications of the subordination principle to univalent functions .......................................... 315

David Sachs, Partition and modulated lattices ..................... 325

Frank S. Scalora, Abstract martingale convergence theorems ............... 347

Elbert A. Walker, Torsion endomorphic images of mixed Abelian groups ........ 375

Morgan Ward, The prime divisors of Fibonacci numbers................. 379

Charles R. B. Wright, On the nilpotency class of a group of exponent four....... 387 